\title{
Software Product Transfers: Lessons Learned from a Case Study
}

\author{
Darja Šmite \\ Blekinge Institute of Technology \\ Karlskrona, Sweden \\ e-mail: Darja.Smite@bth.se
}

\author{
Claes Wohlin \\ Blekinge Institute of Technology \\ Karlskrona, Sweden \\ e-mail: Claes.Wohlin@bth.se
}

\begin{abstract}
Although global software work nowadays is not a phenomenon, research and practice is still addressing the complexities associated with the new forms of work enabled through globalization. 'Go global' strategies usually prescribe two alternative approaches: distribution of software development activities across several locations, or re-location of work to another site. This research paper focuses on the latter approach that is called software product transfers and discusses findings from an empirical case study conducted in Ericsson. The observations indicate that transferring software work from one site to another site of the same company is a challenging endeavor. Furthermore, practices used in colocated projects do not necessarily lead to a desired outcome. The paper provides an overview of the challenges and highlights practical advice for handling software product transfers.
\end{abstract}

Keywords: Global software engineering, global software development, software transfers, distributed teams, offshoring, offshore insourcing, case study

\section{INTRODUCTION}

Companies are becoming more and more multi-national with sites, branches and sub-contractors located in different countries. This is motivated by the intentions to tap into the competence in other countries; reducing costs for development and ensuring proximity to different markets. Globalization in terms of software development has meant that distributed development has become business as usual. Many larger companies, including Ericsson where the case study is conducted, have sites in different countries and hence there is an ambition to maximize the development capability. The latter includes both working jointly on different products across sites and transferring the responsibility and development of products between sites.

The results of global collaborations are reported in very different ways ranging from announcements of tremendous success to total failure. While many companies practice outsourcing of routine tasks and keeping the more interesting work for themselves, offshoring, in particular to India, is often associated with rapid turnover of employees [1]. Therefore many companies are establishing themselves in different countries instead of just relying on outsourcing. The need to ensure that all sites have challenging work and a certain level of responsibility is recognized by the companies. Thus, transfer of work between sites has become a usual business.

Global software development is a major challenge for the software industry and hence also of major interest for researchers. However, most of the papers published are still mainly focused on describing challenges related to globally distributed development [2] and primarily in relation to ongoing development. To the best of our knowledge, research findings related to actual transfer of software work from one site of a company to another site of the same company (offshore insourcing), or a third party (offshore outsourcing) is limited. Due to this matter practitioners are forced to experiment and quickly adjust their tactical approaches for leveraging global software development risks [3].

This paper contributes with a case study related to the actual transfer. The case study has been conducted at a large multi-national telecommunication company, i.e. Ericsson. The study points to a number of challenges when it comes to software product transfers, but also to a set of recommendations to address the challenges faced when transferring a software product from a site in Sweden to a site in India.

\section{RELATED WORK}

The topic of transferring software work is related to studying one specific approach in search of the benefits offered by globalization. It can be observed from either a management perspective addressing decision-making challenges or from an engineering perspective addressing actual execution of a transfer. While "make-or-buy" decisions have been discussed in the research literature for decades [4], the literature focusing on actual transfers is scarce. Some exceptions exist, for example, Mockus and Weiss [5] discuss how to find suitable "chunks" of work for transfer to different sites. They have found that transfer by functionality including the ownership of e.g. a sub-system is the most commonly applied approach in Lucent Technologies [5].

In addition, related research findings from studying initiation and engagement of remote sites and knowledge transfer are helpful for understanding the challenges of 
transferring software work. In the mentioned research [5], the authors point out that the learning curve of the new site takes time due to substantial training required for those who are unfamiliar with the product. Lack of domain and product expertise, in fact, is frequently referred to as one of the key challenges when starting global software development [6], [7], [8].

Knowledge transfer is also associated with a loss in efficiency of developers newly engaged in the project [9]. While companies strive to achieve gains in efficiency by relocating software work to offshore sites, in practice industrial observations suggest that the offshore site can be incapable to fully recover, thus leaving gaps in efficiency after the transfer. The scales associated with evaluating the success of a transfer are illustrated on Figure 1.

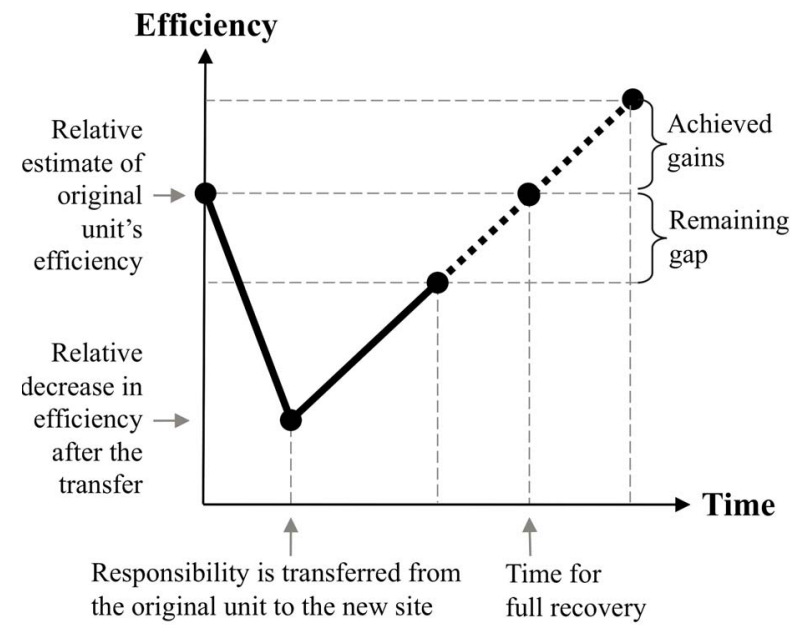

Figure 1. Transfer challenges

Examples of industrial experience provided by Carmel and Tija [9] demonstrate that efficiency can decrease down to $20 \%$ of the relative efficiency of the original unit and rarely achieves full recovery. Overcoming the challenges associated with switching the location of development to another site may also take longer than it takes to meet the objectives on the real capability front [10]. The time for recovery varies from one year for maintenance tasks in $\mathrm{T}$ Corp [9] to more than five years for development in Philips [11]. Other experiences indicate that the learning curve can also be quite challenging after five years of collaboration [12].

It can be concluded that achieving economical success with a transfer of software work is not trivial. This motivates an in-depth analysis of how companies overcome these challenges and ensure minimal decrease in efficiency, and ability of the new site to quickly recover.

\section{RESEARCH METHODOLOGY}

\section{A. Empirical background}

The findings discussed in this paper are based on a case study conducted at Ericsson, a large international company headquartered in Sweden. Ericsson is one of the leading companies in telecommunication market worldwide and is rapidly extending its operation in Asia. The company develops a wide range of products and solutions, including generic software products offered to an open market and complex compound systems with customised versions.

During the past several years Ericsson has transferred a number of software products between different sites. Acknowledging the challenges of software product transfers Ericsson initiated a research project that aims at collecting and documenting experiences for organizational learning. In this research paper the authors present the findings from a single case study.

\section{B. Case study overview}

In order to ease the evaluation of applicability of the findings reported in this paper, the case study is described as suggested by [13].

This study investigates an industrial intra-organizational collaboration between two sites, namely Ericsson in Sweden and India. The case study is a transfer project of a large software component from a product family developed by Ericsson. This product component is particularly interesting due to its history. It has been initially developed by an external software company, which was bought by Ericsson. The component was transferred from the acquired company and it has since been developed in a distributed way. The main responsibility and development has been with Ericsson in Sweden. Ericsson in India has been engaged in product customization tasks. For different reasons it was decided to transfer the responsibility for the entire development of the product component to India. The transfer history is illustrated in Figure 2.

The second transfer project started in January 2009 and was scheduled to finish by November 2009. This project was selected for a case study investigation because it was an on-going transfer with a relative compact schedule that offered an opportunity to observe the entire process of transferring the work.

\section{Research questions}

Motivated by organizational learning objectives the research questions for this case study focused on collecting recommendations for future transfer projects. In particular, the main research questions were as follows:

RQ1: What were the challenges of transferring software work from a site in Sweden to a site in India?

RQ2: Which practices can be applied to mitigate these challenges? 


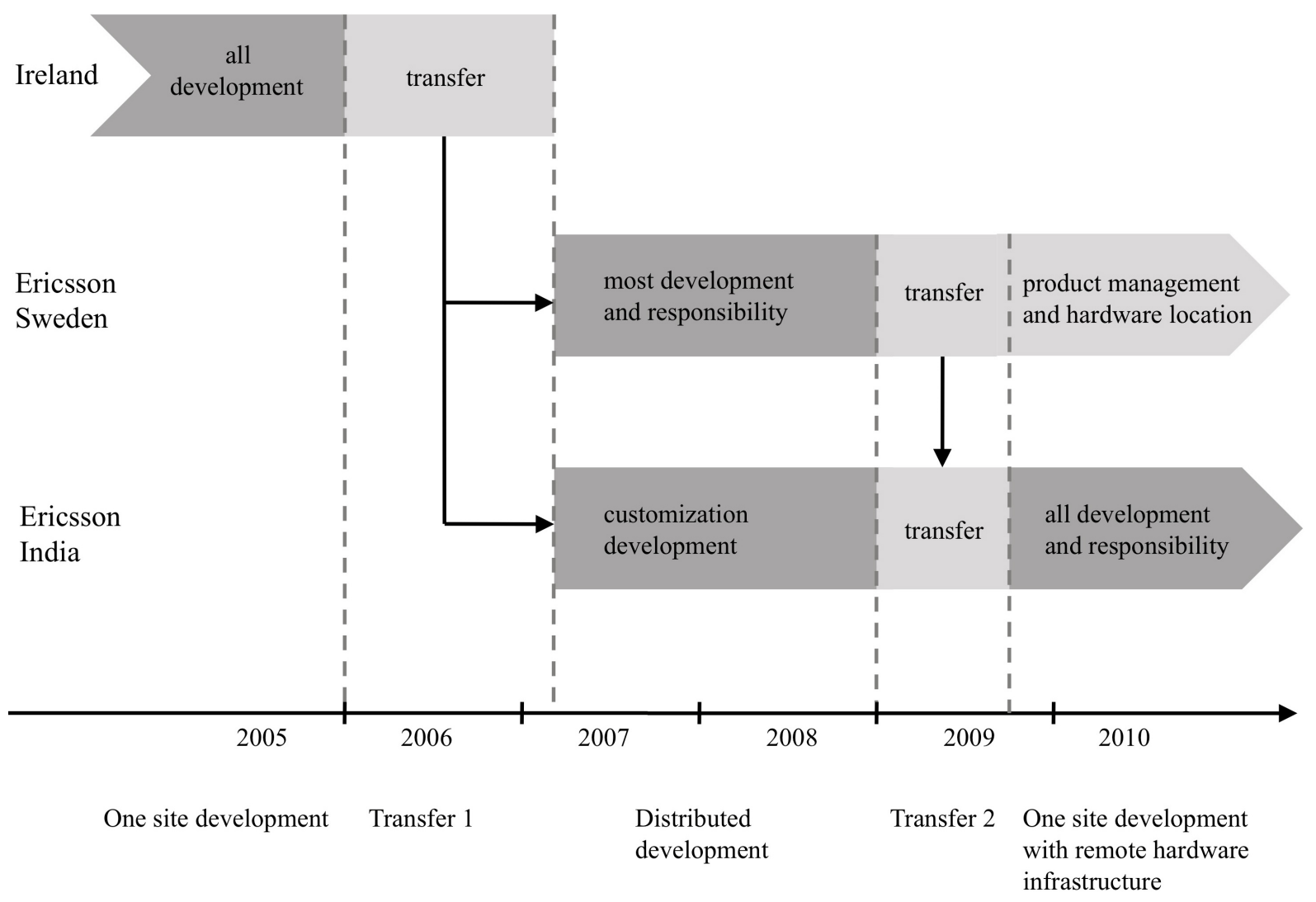

Figure 2. Transfer history

\section{Data collection and analysis}

The research reported is a part of an exploratory study. Herein findings from a single case study are reported based on interviews conducted with Ericsson employees from Sweden and India and an analysis of the transfer project documentation.

The researchers conducted nine interviews in total spread over time of the transfer project. In order to gain different perspectives, employees with different roles were interviewed. All interviews were conducted in Sweden. Therefore it was possible to interview only those Indian employees that were present in Sweden at the time of investigation.

TABLE I. OVERVIEW OF THE INTERVIEWS

\begin{tabular}{lclc}
\hline Site & Time & Roles & No \\
\hline India & Jun & Tech leads & 2 \\
\hline Sweden & Jun & $\begin{array}{l}\text { Development Manager } \\
\text { Tech leads }\end{array}$ & 3 \\
& & Product Managers & 2 \\
\cline { 2 - 4 } & Nov & $\begin{array}{l}\text { Transfer Manager } \\
\text { Development Manager }\end{array}$ & 2 \\
\hline & \multicolumn{2}{c}{ Total number of interviews: } & 9 \\
\hline
\end{tabular}

All interviews were one hour long on average. The conversations were not recorded but documented during the interview and then the notes were sent to the interviewees for approval.

Interview data was uploaded to NVivo 8.0, a special software tool for qualitative data analysis developed by QSR Software. Qualitative analysis techniques as, for example, proposed by Strauss and Corbin [14] were further used to derive transfer challenges and solutions. In particular, open coding was used for labeling different expressions from the interviews and grouping these expressions into related categories (challenges and solutions). Axial coding was then used to identify the relationships between these categories.

In addition, the researchers studied transfer project plans to gain an understanding of the planning and risk management activities, and chronological development of events.

Research findings were first discussed with the management from the case study project. Email interaction was used to finalize the findings and ensure the accuracy of the case history. The findings were then presented to the research stakeholders at a monthly project reference group meeting. 


\section{FINDINGS}

The observations from the case study suggest that the process of transferring software work from Sweden to India has undergone two phases:

1. The first phase was focused on building the receiving site (in India): ensuring the resources and their competence. Meanwhile the sending site (in Sweden) was dominant in the on-going development.

2. In the second phase the receiving site is handing the development while the sending site is still providing active coaching and support.

At the end of the transfer project the receiving site shall be capable of handling the development without coaching and support, and hence the responsibility of the sending site is cut off. The dynamics of activities between the involved sites are illustrated in Figure 3.

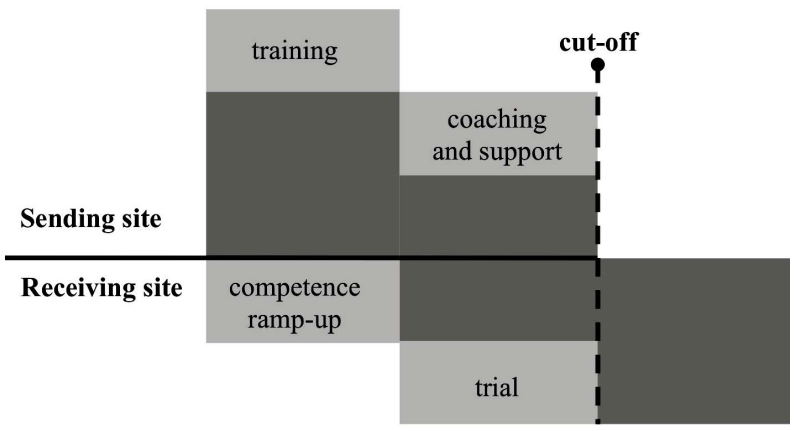

On-going product development

Transfer project activities

Figure 3. Transfer activities and on-going development

In the following sub-sections, observations and lessons learned from the case study are presented. The findings are organized as a set of challenges faced during the transfer and solutions that were applied to mitigate these challenges. Quotes from the interviews are used to illustrate the atmosphere in the investigated case.

\section{A. Finding the right people}

Ability to find the right people with the right competence when building the receiving site is crucial. While finding resources in general for the receiving site may seem a relatively easy task, in particular considering the assumed opportunities offered by the Indian human resource market, the studied case suggests that recruitment of resources for specific roles with specific competence at a specific point in time may be difficult.

According to a Tech Lead from India, resources are the number one challenge. He stressed that finding a 1:1 mapping to replace the resources from the sending site with those at the receiving site is challenging because recruitment in India is tough nowadays.
Despite all "head-hunting" activities from human resource management, the studied transfer project was unable to recruit some key personnel with the right competence for some of the leading positions.

Solution: The recruitment process shall start early, i.e. before starting the actual transfer. In case, recruitment becomes a challenge, organizations may turn to an alternative solution. Instead of struggling with the recruitment process, the studied organization changed the strategy to promoting existing employees into the core team and recruiting new employees for positions related to development and testing. The downside of this approach however was related to delayed competence transfer and additional training that was required as a consequence of promoting existing employees. On the other hand, Ericsson succeeded to reduce the turnover of their employees in India from $11 \%$ in 2008 to $4 \%$ in 2009 by engaging them in more challenging roles over time.

\section{Recommendation: Recruit people before the transfer}

\section{B. Transferring the competence}

After getting the resources in place it is essential to ensure the capability of the receiving site to run the development after the transfer is finished. This prescribes transferring the product competence from experienced sending resources to the receiving resources that are less experienced and in particular have less product knowledge.

Observations indicate that a list of factors determine how successful the competence transfer will be. The main factors are related to people experience and expertise, product documentation, training approaches and availability of resources for the after-support.

1) Dealing with implicit knowledge: As already mentioned, expertise plays the key role. However, previous experience with the product is even more important. This is not only related to the technical knowledge, but also the history of the product itself that is often implicit. Several interviewees indicated that it is impossible to support a product without a complete documentation of decisions made during the product development. The decisions may for example relate to certain architectural decision. These, in fact, may not even be logical or obvious, but due to certain reasons have been selected as being most effective for the product at a specific point in time. Ways of working is another example of implicit knowledge that is difficult to transfer.

A Tech Lead from Sweden emphasized that it's hard to transfer experience, when it comes to explaining how one reacts in different situations, and what decisions are taken and sometimes also why certain solutions were chosen. 
Solution: One of the solutions that has been recognized as a best practice at Ericsson for overcoming the consequences of cutting the product history with the transfer prescribes transferring people with the product. Employees from the sending site are encouraged to move to the receiving site at least for a year or two. The positive effect of this practice has been noticed in other product transfers and was specifically addressed when the studied transfer project was planned. In spite of pessimistic expectations from the transfer project management six employees were ready to follow the product to India.

\section{Recommendation: Transfer people with the product}

2) Effective training approaches: Transferring people with the product however is not a long-term solution. The sending site shall find a good way to transfer the core competence and train the receiving site in handling product development. This nonetheless is not always a straightforward task. The observations indicate that some training approaches that are useful in Sweden did not work in India.

According to a Development Manager from Sweden, the learning process can be affected by cultural differences. He noted that while in Sweden people are expected to demonstrate initiative in finding solutions on your own and it is common to learn by mistakes, in other cultures people are used to be guided and may lose their face if making mistakes.

Solution: Interviewees from the studied case emphasized that knowledge needs to be transferred from person to person. Although it has been noted that global teams rarely meet due to high traveling costs, Ericsson best practices prescribe on-site co-located training for weeks or even months (up to half a year) to ensure efficient competence transfer.

But co-location as such is also not a long-term solution. The interviewees emphasized, that it is easy to waste the time spent in the receiving site if not well planned in advance. The experience also shows that competence transfer through documentation and theoretical training even in co-location is inefficient. Different training approaches have been tried out, but both sites recognized hands-on practical training as the most effective solution.

A Tech Lead from Sweden indicated that engaging the receivers in the daily work is the only way to learn how to bring the quality into the product and continue to maintain the quality after the transfer.

Recommendation: Plan co-located hands-on training
3) Limiting the scope: Interviewees argue that transfers are never complete. It is impossible to transfer $100 \%$ of the knowledge about the product, or go through a complete training that covers every single situation. The interviewees note that some particular problems may take several years to experience and no training program can cover all possible situations.

Solution: Because of these challenges and tight transfer project schedules, it is therefore suggested to focus on the core team, core roles, and core documentation. While this may seem obvious, the observations stress the need for a proper plan, otherwise there is a clear risk that it may not happen due to other commitments.

\section{Recommendation: Focus on key roles/items}

4) A helping hand: When the cut-off date comes, the receiving site finally receives the responsibility and their capabilities will be tested in real-life circumstances. The case study indicates that there will be confusion and maybe even panic. This is natural. A key question is whether this process will affect the product and existing customers. Interviewees' reflections on the previous transfers suggest that when the transfer project comes to an end, the sending resources are often moved to other projects and can rarely support the receiving site.

Solution: Lessons learned in previous transfer cases suggested to organize a trial period while still having the sending site available for coaching and support. In this way, the responsibility was handed over before the sending resources were released.

\section{Recommendation: Trial before the cut-off}

Solution: The interviewees noted that the main emphasis during this time should be put on coaching rather than helping by doing. Although it is easier and faster to do things yourself it will not increase the abilities or decrease the insecurities of the new product developers.

\section{Recommendation: Support through coaching}

5) Motivation: It is worth mentioning that sending resources play a vital role in transferring the knowledge. Since transfers are often associated with fear and uncertainty about employees' future, it has been noted that this might lead to a lack of interpersonal trust between people at onsite and offsite [15]. 
Solution: In the studied case the transfer was initiated to free-up resources and the new assignments for the sending resources were announced together with the transfer decision. This ensured the motivation of those involved in the knowledge transfer.

\section{Recommendation: Ensure motivation}

6) Documentation: Although it is impossible to document all the necessary instructions on handling product development, documentation does matter.

Solution: Good documentation ensures easier maintainability and relieves the pressure from the sending site after the transfer is finished. Transfer project management in the investigated case decided to compensate the gaps in documentation, which resulted in the effort of one consultant employed full time for nine months.

Recommendation: Ensure product documentation

7) Maintaining on-going development: Due to the market pressure a software product driven company nowadays cannot allow any product to be out of business for any longer time. This is why the transfers are required to be balanced with the on-going development as shown on Fig. 2. The employees from the sending site reported that getting the everyday job done while being engaged in coaching and training activities is challenging.

A Development Manager from Sweden stressed that running active development at the same time with the transfer is a challenge. He explained that a large number of customers demand their needs to be addressed immediately. Therefore, having continuous capacity and preventing the negative effect of a transfer becomes a must and the main focus of the management.

It is worth emphasizing that many other employees mentioned that the demand of high-level management to have no impact during and after a transfer is not really realistic.

A Product Manager from Sweden mentioned that, despite the good will and management requirements, everybody knows that transfers come with certain consequences.

Solution: The observations together with experience reported from other product transfers suggest that transfers definitely impact the on-going development activities. The same people cannot continue developing the product with the same capacity while coaching and training the receiving site. To alleviate the transfers, Product Managers suggest reducing the amount of requirements in the product releases that are developed in parallel with the transfer.

Recommendation: Balance on-going development with the transfer

Solution: Additionally, the employees at the receiving site advise that things shall not be pushed or rushed. A transfer requires time and it is better to approach transfers step-by-step, not overnight. For example, in the studied case the receiving site was gradually involved into product customization and maintenance tasks before the actual transfer of the product responsibility was performed. Although this means that the duration of a transfer project is longer, experience shows that the negative effect on the ongoing development during and after the transfer can as a result be reduced.

\section{Recommendation: Transfer activities step by step}

\section{Overcoming cultural differences}

All interviewees from Sweden have noted the importance of acknowledging and understanding the cultural differences. In addition to the cultural misunderstandings when people say the same things with different meanings, which in fact become clichés, the effect of cultural differences is not always easy to evaluate.

A Tech Lead from Sweden said that although the cultural differences are now known, the consequences and required actions are not fully realized. He also emphasized that because of cultural differences corrective actions may not always work.

One of the challenges as pointed out earlier relates to how people from different cultural backgrounds learn. The interviewees from the investigated transfer project state that there were misunderstandings due to unreasonable expectations that unavoidably caused time delays and required changing the strategies.

Solution: Several interviewees noted that things could have been much easier if the cultural differences were known in advance. Thus, cultural awareness is regarded as one of the important skills. A new Cross-Cultural Communication Course is organized for transfer project employees in Ericsson. The course brings together employees from the collaborating sites and opens up a dialog about their diversity. It is worth emphasizing that it is important to maintain a mixture of sending and receiving employees to avoid dominance of the overly represented site and protectiveness of the other site. The benefit of attending this course has been acknowledged and it is further suggested to 
be a mandatory practice not only for managers, but also for the entire transfer team.

\section{Recommendation: Train cultural awareness early}

\section{DISCUSSION}

\section{A. Monitoring and measuring transfers}

While analyzing success or failure of a transfer, the authors made an interesting observation: a transfer is a separate project that is not measured according to the same factors as the development project. The studied company measures the budget and schedule of a transfer project, and its effect on on-going development. The transfer project is finished, when the receiving site takes over the main responsibility for the development. No further measures are collected in relation to the consequences of a transfer. This means that a successful on-time within-budget transfer that did not interfere with the on-going development is successful in spite of the further effect on the efficiency and quality. However, if the Product Managers slow down the development during the transfer, it is difficult to capture the effect of the transfer with traditional measures since the amount of developed requirements is reduced.

The transfer project reported here does not yet allow evaluating the further effect of the transfer on the product. Nonetheless, some of the interviews indicate that careful attention shall be paid on monitoring the life of the product after the transfer is finished. Looking at a transfer project, it is possible to observe that in the beginning there are certain risks that over time may turn into immediate effect (e.g. additional transfer costs as in the studied case). When the transfer project is finished the next wave of consequences may appear. This is expected to have an effect on the product and after-transfer development (see Figure 4).

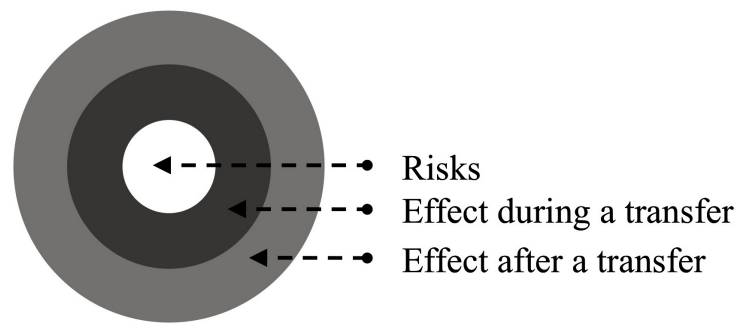

Figure 4. The effect of a transfer

The studied case succeeded in addressing many of the initial risks that did not become a problem during the transfer. Observations illustrate activities that were taken and suggest that it is possible to accept certain risks, however with certain costs.

Other effects will be possible to evaluate only after a certain amount of time of development at the receiving side. One of the main concerns in this respect is product quality.
A Tech Lead from Sweden said that having experience allows improving the product. He argued that a product that is moved around would never get into a state where the product will continuously improve.

It is worth mentioning, that the responsibility for measuring the product status after the transfer shall be discussed and assigned in advance since many stakeholders may leave the product after the transfer. In the studied case the Transfer Manager and the Development Manager were assigned to other products when the transfer was completed, while Product Managers responsible for product evolution will continue their involvement.

\section{B. Selecting candidates for a transfer}

An important question is whether any product really is suitable for a transfer. The studied case suggests that the answer is no. The majority of transfers are motivated by economical reasons. This means, that a transfer of a product shall be economically viable. Unfortunately, a transfer is a costly process itself. The studied transfer project reported additional costs for developing product documentation, retraining of the promoted employees, prolonging of the sending resources for a trial period and after-transfer support, and finally slowing down the development by decreasing the throughput in order to smoothen the consequences of the transfer.

These are natural consequences of any transfer that shall be taken into consideration. The findings suggest that a product with a short life cycle is therefore not suitable for a transfer.

A Product Manager estimated that a full transfer (end of all questions to the sending site) takes five to six years, and therefore transfer decisions shall have intentions for at least a seven to eight years long period to allow reaching the economic benefits.

Besides these reasons, lessons learned from the case study suggest that some products are easier to transfer. Therefore, it is recommended to consider the following factors:

- Complexity and maturity of the product,

- Product documentation,

- Previous experience with the product on the receiving site.

\section{IMPLICATIONS FOR RESEARCH AND PRACTICE}

The reported findings are based on a single case study conducted in one company that transfers software work between a site in Sweden and a site in India. However, the recommendations have been discussed with other managers who have been involved in other transfers. They acknowledge the recommendations provided here as being important in successfully transferring software products between sites within a company. Thus, the given recommendations are expected to be valid more generally than for the specific case. The recommendations should be of 
value for software companies (at least as a starting point for being successful in transfer initiatives) involved in transferring work between geographically, temporally and culturally distant sites of the same company.

This study exemplifies the challenges that may be faced while transferring software work between two sites located in different countries, in different parts of the world with time differences and cultural differences. It serves as a building block towards an understanding of the nature of software product transfers and contributes with practical recommendations for overcoming the identified challenges.

The findings suggest that future research shall focus on providing a better support for offshore decisions, in particular through quantitative evaluation and empirical evidence from different companies, locations and transfer strategies.

Practical implications of this study suggest that, when a company considers transferring software work to another site, management shall pay deserved attention to selecting a product feasible for a transfer, planning the duration and approach of the transfer, balancing the transfer with on-going development activities, and ensuring after-support performance.

\section{CONCLUSIONS}

Transferring software work across geographically, temporally and culturally distant sites even within one company is not a straightforward task. Related research indicates that transfers are often associated with decreased productivity and lengthy recovery. Potential challenges discussed in this paper provide a deeper understanding of the reasons behind efficiency problems, highlighting project staffing, knowledge transfer, continuity of ongoing development, and cultural awareness as the key ones. Although some of the given recommendations may seem obvious, practitioners from the studied organization indicate that these practices have emerged from experience with the previous transfers, and have been initially overlooked.

Nonetheless, case observations suggest that if addressed, these challenges can be mitigated. The following practical recommendations that can be utilized for transfer project planning were found useful in the studied company:

\section{Before-transfer activities}

- Recruit people before the transfer. Searching for new employees with the right competence to fill the gaps during the transfer is too late and might endanger the deadlines and cause ineffective usage of time allocated for experience sharing.

- Focus on key resources/items. Transfers are never complete and it will not be feasible to teach everybody, or provide training for every possible task. Therefore, a core team and core tasks shall be selected and prioritized.

- Plan the transfer activities step by step. Similarly to iterative development, iterative transfer of work is recognized as more effective because it is easier to transfer clearly defined chunks with foreseeable schedules.

- Balance on-going development with the transfer. Training and coaching requires time and shall be prioritized. Thus, people involved in the transfer shall be provided with sufficient independence from the ongoing development tasks. Even if this means reducing the amount of development tasks.

- Motivate people who transfer the knowledge. Employees who are confused or threatened to loose their jobs will likely demonstrate non-cooperative behavior with their receiving counterpart. To avoid this, a clear vision of their future shall be communicated early for motivation.

- Train cultural awareness early. Culture has an impact on how people behave, learn, work, teach, etc. Therefore, selection of approaches shall be culturally informed.

\section{Transfer activities}

- Organize co-located hands-on training. Knowledge shall be transferred from person to person. Pair people and let them share experiences. Note that learning from documentation is recognized as ineffective; learning by doing strengthens the understanding of tasks through gaining practical experience.

- Ensure product documentation. Although it is not possible to document everything, core documentation will ensure continuity of previous development traditions and more independence of the new product team.

- Trial before the cut-off. This ensures the necessary experience for the new product team while being in a safety net of the sending resources.

\section{After-transfer activities}

- Transfer people with the product. Not all knowledge can be taught or documented. Keeping experts with the product alleviates contingency of the quality.

- Support through coaching. While experienced developers can resolve any problem within a short time on the behalf of the new product team, this will not support the learning.

Furthermore the findings suggest that a transfer does not end when the transition of product development is finished. A transfer can have immediate and long-term consequences. Therefore support and monitoring shall be continuously maintained to ensure efficiency and product quality.

In addition, the observations advocate that success of a transfer is also dependent on informed selection of the product for transfer.

As a future research direction we aim to quantify the costs associated with transferring software work from one location to another, to understand the feasibility of transferring software products. We would also like to explore the benefits of the Handshaking approach [16] that promises transferring and providing a learning effect across 
organizational units with regards to engineering tradition, technical knowledge, and cultural homogenization.

\section{ACKNOWLEDGEMENTS}

We would like to thank Ericsson employees for their interest, active participation and support of this research.

The research is funded by Ericsson Software Research and the Swedish Knowledge Foundation under the KK-Hög grant 2009/0249.

\section{REFERENCES}

[1] Conchuir E.O, H. Holmström, P.J. Ågerfalk and B.Fitzgerald, "Exploring the Assumed Benefits of Global Software Development", In: Proceedings of the 1st International Conference on Global Software Engineering, 2006, pp.159-168

[2] Smite D., C. Wohlin, R. Feldt, and T. Gorschek "Empirical Evidence in Global Software Engineering: A Systematic Review". In: the Journal of Empirical Software Engineering, 15(1), 2010, pp.91-118

[3] Carmel, E. and Agarwal, R. "Tactical Approaches for Alleviating Distance in Global Software Development” In: IEEE Software, 18(2), 2001, pp.22-29

[4] Lee, J.-N., Huynth M.Q., Kwok R.C.-W., and Pi S-M., "IT Outsourcing Evolution - Past, Present, and Future", Communications of the ACM, 46 (5), 2003, pp.84-89

[5] Mockus A. and D.M. Weiss, "Globalization by Chunking: A Quantitative Approach", In: IEEE Software 18(2), 2001, pp. 30-37

[6] Herbsleb J.D., D.J. Paulish, and M. Bass, "Global software development at Siemens: Experience from nine project", In Proceedings of the 27th International Conference on Software Engineering, 2005, pp. 524-533
[7] Smite D., "Global software development projects in one of the biggest companies in Latvia: Is geographical distribution a problem?", Journal of Software Process Improvement and Practice, 11 (1), 2006, pp. 61-76

[8] Battin R.D., R. Crocker, J. Kreidler, and K. Subramanian, "Leveraging resources in global software development", IEEE Software, 18(2), 2001, pp. 70-77

[9] Carmel E. and Tjia, P., "Offshoring Information Technology: Sourcing and Outsourcing to a Global Workforce". Cambridge University Press, NY, 2005

[10] Banerjee, A., and Williams, S.A., "Using offshore analytics to identify determinants of value-added outsourcing". In: Strategic Outsourcing: An International Journal, 2(1), 2009, pp. 68-79

[11] Kommeren, R., and Parviainen, P., "Philips experiences in global distributed software development", In: Empirical Software Engineering, 12 (6), 2007, pp.647-660

[12] Boden A., Nett, B. and Wulf, V. "Coordination Practices in Distributed Software Development of Small Enterprises", In Proceedings of the 2nd International Conference on Global Software Engineering, 2007, pp.235-246

[13] Smite D., C. Wohlin, R. Feldt, and T. Gorschek "Reporting Empirical Research in Global Software Engineering: a Classification Scheme", In: Proceedings of the 3rd International Conference on Global Software Engineering, 2008, pp. 173-181

[14] Strauss A, J. Corbin, Basics of Qualitative Research: Techniques and Procedures for Developing Grounded Theory, Sage Publications: Thousand Oaks, CA, 1998

[15] Piri A., T. Niinimäki, and C. Lassenius, "Descriptive Analysis of Fear And Distrust in Early Phases of GSD Projects". In: Proceedings of the 4th International Conference on Global Software Engineering, 2009, pp. $105-114$

[16] Fricker S., Gorschek T., Byman C., and A. Schmidle, "Handshaking: Negotiate to Provoke the Right Understanding of Requirements", In: IEEE Software, 27(2), 2010, pp.72-80 